\title{
Renewal, modulation, and superstatistics in times series
}

\author{
Paolo Allegrini, ${ }^{1,2}$ Francesco Barbi, ${ }^{2}$ Paolo Grigolini, ${ }^{2,3,4}$ and Paolo Paradisi ${ }^{5}$ \\ ${ }^{1}$ Istituto Nazionale di Fisica della Materia, unità di Como, Via Valleggio 11, 22100 Como, Italy \\ ${ }^{2}$ Dipartimento di Fisica "E.Fermi"-Università di Pisa, Largo Pontecorvo, 356127 Pisa, Italy \\ ${ }^{3}$ Center for Nonlinear Science, University of North Texas, P.O. Box 311427, Denton, Texas 76203-1427, USA \\ ${ }^{4}$ Istituto dei Processi Chimico Fisici del CNR, Area della Ricerca di Pisa, Via G. Moruzzi, 56124, Pisa, Italy \\ ${ }^{5}$ ISAC-CNR, Sezione di Lecce, Strada Provinciale Lecce-Monteroni km 1.2 I-73100 Lecce, Italy
}

(Received 17 March 2005; revised manuscript received 19 December 2005; published 27 April 2006)

\begin{abstract}
We consider two different approaches, to which we refer to as renewal and modulation, to generate time series with a nonexponential distribution of waiting times. We show that different time series with the same waiting time distribution are not necessarily statistically equivalent, and might generate different physical properties. Renewal generates aging and anomalous scaling, while modulation yields no significant aging and either ordinary or anomalous diffusion, according to the dynamic prescription adopted. We show, in fact, that the physical realization of modulation generates two classes of events. The events of the first class are determined by the persistent use of the same exponential time scale for an extended lapse of time, and consequently are numerous; the events of the second class are identified with the abrupt changes from one to another exponential prescription, and consequently are rare. The events of the second class, although rare, determine the scaling of the diffusion process, and for this reason we term them as crucial events. According to the prescription adopted to produce modulation, the distribution density of the time distances between two consecutive crucial events might have, or not, a diverging second moment. In the former case the resulting diffusion process, although going through a transition regime very extended in time, will eventually become anomalous. In conclusion, modulation rather than ruling out the action of renewal events, produces crucial events hidden by clouds of exponential events, thereby setting the challenge for their identification.
\end{abstract}

DOI: 10.1103/PhysRevE.73.046136

PACS number(s): 05.65.+b, 05.40.Fb

\section{INTRODUCTION}

The new field of complexity is attracting the attention of an increasing number of researchers, and it is triggering vivacious debates about its true meaning [1-3]. Here we adopt the simple minded definition of complexity science, as the field of investigation of multi-component systems characterized by noncanonical distributions. On intuitive ground, this means that we trace back the deviation from the canonical form of equilibrium and relaxation to the breakdown of the conditions on which Boltzmann's view is based: Short-range interaction, no memory, and no cooperation. Thus, the deviation from the canonical form, which implies total randomness, is a measure of the system complexity.

However, this definition of complexity does not address the delicate problem of the origin of the departure from canonical distributions. We plan to discuss this issue, although focusing our attention on waiting time rather than energy distribution. Thus, the canonical case is represented by the waiting time exponential distribution and the noncanonical condition is given by a distribution of waiting times that in the time asymptotic limit gets the form of an inverse power law. We use two different approaches to the time series $\left\{t_{i}\right\}$, so as to ensure, however, that in both cases the distribution density of the time distances $\tau_{i}=t_{i+1}-t_{i}$ is

$$
\psi(\tau)=(\mu-1) \frac{T^{\mu-1}}{(\tau+T)^{\mu}},
$$

with $1<\mu<\infty$. Note that the parameter $T>0$, which eliminates the unphysical divergence at $t=0$, gives also informa- tion on the lapse of time necessary to reach the time asymptotic condition where $\psi(\tau)$ becomes identical to an inverse power law. The choice of the form of Eq. (1) is dictated by the simplicity criterion. This form has been known for many years [4] see, for instance, Ref. [5], and following Metzler and Nonnenmacher [4] and Metzler and Klafter [6] we shall be referring to it as Nutting law.

The two different approaches that we use to generate the time series $\left\{t_{i}\right\}$ and the corresponding waiting time distribution of Eq. (1) are dynamic and are based on the renewal and modulation perspective. The renewal approach generates the time series $\left\{t_{i}\right\}$ by means of dynamical processes that reset the system's memory to zero after the production of each waiting time $\tau_{i}$. Thus, the renewal approach to $\left\{t_{i}\right\}$ is equivalent to drawing the numbers $\tau_{i}$ from the distribution of Eq. (1), with each drawing having no memory of the earlier drawings. The modulation approach, on the contrary, is based on a dynamic process that for a very extended period of time generates exponentially distributed waiting times with a fixed exponential scale. This dynamic process is realized in this paper with two different prescriptions, and with both prescriptions, from time to time a transition from a given exponential scale to another occurs, in such a way that the resulting waiting time distribution, which is, therefore, the superposition of infinitely many exponential distributions, gets the form of Eq. (1). In conclusions, these two approaches, although different, lead to the same nonexponential distribution of Eq. (1). Thus, to a first sight, one might be tempted to conclude that they are indistinguishable, leaving no motivation whatsoever to prefer the one to the 
other. We shall prove that it is not so, and that there exist physical properties, whose observation allows us to distinguish the two proposals and to assess which one is correct for the given complex process under consideration.

The first property is renewal aging [7-9]. In an earlier work [10] the conjecture was made that infinitely slow modulation does not yield aging, while the renewal models do. In this paper we check this prediction by means of numerical simulation, and, as earlier mentioned, we create artificial time series, according to the modulation and the renewal prescription, and we find that the conjecture of Ref. $[10]$ on the lack of aging in the case of infinitely slow modulation, is correct. As a second property to explore, to distinguish modulation from renewal, we select diffusion. To generate diffusion we proceed as follows. We note that between an event occurring at time $t_{i}$ and the next, occurring at time $t_{i+1}>t_{i}$, there is an empty time interval that we call laminar region. The reason for the choice of this term is that the dynamical model that we use to realize the renewal condition is an idealization of the Manneville map [11], which has been originally used to study fluid-dynamic turbulence. This term is appropriate, in principle, only within the renewal perspective. However, for simplicity we shall use the term laminar region, regardless of whether we work with the modulation or the renewal model. Then for each laminar region we assign a constant value, either $W$ or $-W$, according to a fair coin tossing prescription, to a velocity variable $\xi(t)$. In other words, we convert the time series into a dichotomous fluctuation $\xi(t)$, and we use this fluctuation to generate the diffusion of the space coordinate $x$ according to the usual prescription

$$
x(t)=\int_{0}^{t} \xi\left(t^{\prime}\right) d t^{\prime}
$$

Thus, we are now in a position to illustrate the second property that we shall explore to distinguish modulation from renewal. This is diffusion scaling. The diffusion process generated according to the earlier prescription is described by the probability density function (PDF) $p(x, t)$. In the asymptotic regime the PDF is expected to yield the scaling property

$$
p(x, t)=\frac{1}{t^{\delta}} F\left(\frac{x}{t^{\delta}}\right),
$$

with the parameter $\delta$ called scaling coefficient. The departure from ordinary statistical mechanics is signaled by either $\delta \neq 0.5$ or $F(y)$ different from the Gaussian form, or by both properties. In the renewal case the scaling parameter $\delta$ is determined by the waiting time distribution of Eq. (1). In the case $2<\mu<3$ it is well known see, for example, [12,13], that

$$
\delta=\frac{1}{\mu-1} .
$$

Note that the waiting time distribution of Eq. (1), whose power index $\mu$ is adopted to result in the scaling prediction of Eq. (4), can be derived, in principle, from the experimen- tal observation, by converting into a histogram the set of time distances between consecutive events. In the case of modulation we shall find the interesting result that the scaling coefficient is not determined by the power index of (1). This means that it is not determined by the experimental histogram. This is so because the scaling coefficient is determined by the events corresponding to the abrupt change of exponential scale. If the modulation is slow, these events are very rare, but have nevertheless the important effect of determining the scaling of the diffusion process. For this reason we call these events crucial events. The set of time distances between two consecutive crucial events, converted into a histogram, generates the waiting time distribution $\psi_{\text {crucial }}(\tau)$. The scaling of the diffusion process generated by modulation depends on $\psi_{\text {crucial }}(\tau)$ rather than $\psi(\tau)$, and it yields the same scaling as renewal theory, only if the power index of $\psi_{\text {crucial }}(\tau)$ is the same as that of $\psi(\tau)$. The ratio of the number of crucial events to the total number of events tend to zero when modulation becomes infinitely slow, this being the condition considered in the earlier work [10]. In this paper we shall study the case when modulation is not infinitely slow, so that the scaling determined by the crucial events is produced in a finite time.

In Secs. II and III we explain how to generate the nonexponential waiting time distributions according to renewal and modulation prescriptions, respectively. Thus, these sections give also a more precise definition of modulation and renewal. In Sec. IV we shortly review the concept of aging and we illustrate an aging experiment that turns out to afford an efficient criterion to distinguish modulation from renewal, in accordance with the conjecture of the earlier work of Ref. [10] and with the results of a more recent paper [14]. Section V is devoted to studying the diffusion process generated by either renewal or modulation, with the interesting discovery that the physical realization of modulation might generate renewal properties. With Sec. VI we reiterate the importance of crucial events, which, although ostensible in the case of renewal theory and virtually invisible in the case of modulation, are responsible for diffusion scaling in both cases.

\section{RENEWAL}

We think that a reliable prototype of renewal process is afforded by a dynamical model used in earlier work [9,15-18]. To make this paper as self-contained as possible, let us review here this simple model. Let us consider a particle moving within the interval $I=(0,1]$ driven by the following equation of motion

$$
\frac{d}{d t} y=a y^{z}
$$

with

$$
z \geqslant 1
$$




$$
a>0 \text {. }
$$

Due to the positivity of $a$ the particle moves from the left to the right, and any time it reaches the border $y=1$ is injected back to a randomly chosen position $y_{0}$, meeting the condition

$$
0<y_{0}<1
$$

The distribution density of sojourn times, $\psi(\tau)$, is evaluated as follows. First of all, we solve Eq. (5) to determine the time necessary for the particle to reach the border moving from a given initial condition $y_{0}$. This time is given by

$$
\tau=\frac{1}{a(z-1)}\left[\frac{1}{y_{0}^{z-1}}-1\right] .
$$

The probability for the particle to get the border in the infinitesimal interval $[\tau, \tau+d \tau]$ is determined by

$$
\psi(\tau) d \tau=p_{0}\left(y_{0}\right) d y_{0} .
$$

We make the assumption of uniform back injection, which yields $p_{0}\left(y_{0}\right)=1$. Thus, we obtain the form of Eq. (1) with

$$
\mu=\frac{z}{z-1}
$$

and

$$
T=\frac{\mu-1}{a} .
$$

It is interesting to notice that using Eq. (1) leads to the following expression, for the mean sojourn time $\langle\tau\rangle$,

$$
\langle\tau\rangle=\frac{T}{\mu-2} .
$$

Thus, using Eq. (12) we express $\mu$ as follows

$$
\mu=1+a T=2+\frac{T}{\langle\tau\rangle} .
$$

The condition $p_{0}\left(y_{0}\right)=1$ is the initial distribution of the variable $y$. Upon change of time this distribution tends to become peaked around $y=0$, and in the case where $\mu>2$, it is shown [17] to reach the invariant distribution $p_{e q}(y)=(2-z) / y^{z-1}$. The formula of Eq. (14) refers to the case when the invariant distribution exists, as made evident by the fact that $\langle\tau\rangle<\infty$, thereby yielding $\mu>2$. This is the condition considered in this paper.

In practice, we create first the sequence $\left\{y_{0}(i)\right\}$, by means of a succession of random drawings of numbers from within the interval $I$. Then, using the transformation of Eq. (9), we associate $y_{0}(i)$ with $\tau_{i}$, thereby creating the sequence $\left\{\tau_{i}\right\}$. As mentioned in Sec. I, we use the time series $\left\{\tau_{i}\right\}$ to create the time series $\left\{t_{i}\right\}$ : The first event occurs at $t_{1}=\tau_{1}$, the second at time $t_{2}=\tau_{1}+\tau_{2}$, and so on.

The renewal character of the model is made evident by Eq. (9). In fact, the values of $y_{0}$ are randomly chosen from a uniform distribution, $0<y_{0}<1$. Any drawing does not have memory of the earlier drawings. Consequently, a laminar re- gion does not have any memory of the earlier laminar regions. In literature there are many examples of renewal models yielding the distribution of Eq. (1). Here we quote first the model illustrated by Zaslavsky [19], known as the hierarchical island trap model. In the specific case of blinking quantum dots, a model of the same kind as that of Ref. [19] has been proposed by the authors of Ref. [20]. This model, in turn, is formally equivalent to that proposed years ago by Bouchaud $[21,22]$, to explain the dynamics of glassy systems. In this paper, to work within the renewal perspective we use the dynamical model of this section, which, although very simple, is essentially equivalent to the models currently adopted to produce non-Poisson renewal processes $[19,21,22]$.

\section{MODULATION THEORY}

To illustrate the modulation approach to the noncanonical distribution of waiting times, let us consider a symmetric double-well potential under the influence of white noise, yielding the exponential distribution of the time of sojourn in the two wells [23]. We have

$$
\psi(t)=\lambda \exp (-\lambda t)
$$

The parameter $\lambda$ is determined by the Arrhenius formula

$$
\lambda=k \exp \left(-\frac{Q}{k_{B} \theta}\right) .
$$

In the case when either the barrier intensity $Q$ [23] or temperature $\theta[24]$ are slowly modulated, the resulting waiting time distribution becomes a superposition of infinitely many exponentials. At least since the important work of Shlesinger and Hughes [25], and probably earlier, it is known that a superposition of infinitely many exponentially decaying functions can generate an inverse power law. This, by itself, does not qualify the theory as modulation. It depends on the criterion adopted to relate the time series $\left\{\tau_{i}\right\}$ to the time series $\left\{t_{i}\right\}$. Let us consider, for instance, the time $t_{i}$, and let us imagine that an exponential distribution with time scale $1 / \lambda_{i}$ is used to draw the time distance $\tau_{i}$. This allows us to define the time $t_{i+1}=t_{i}+\tau_{i}$. Let us now move to define time $t_{i+2}=t_{i+1}+\tau_{i+1}$, with the condition that $\tau_{i}$ is drawn from an exponential distribution with time scale $1 / \lambda_{i+1}$, with $\lambda_{i+1}$ not correlated to $\lambda_{i}$. The resulting process is no doubt renewal. To produce modulation, we have to use the same exponential distribution for a large number $N_{d}$ of consecutive drawings.

In recent times, the term superstatistics has been coined [26] to denote an approach to noncanonical distributions, of any form, not only the Nutting form, as in the original work of Beck [27]. We note that Cohen points out explicitly [26] that the time scale to change from a canonical distribution to another must be much larger than the time scale of each canonical process. Thus, we can qualify superstatistics as a form of slow modulation. Therefore, from now on we shall indifferently refer to this approach to complexity either as slow modulation or superstatistics. 
In conclusion, according to the modulation theory we write the waiting time distribution $\psi(t)$ under the following form

$$
\psi(\tau)=\int d \lambda \Pi(\lambda) \lambda \exp (-\lambda \tau)
$$

where $\Pi(\lambda)$ is the $\Gamma$ distribution of order $\mu-1$ given by

$$
\Pi(\lambda)=\frac{T^{\mu-1}}{\Gamma(\mu-1)} \lambda^{\mu-2} \exp (-\lambda T) .
$$

This formula, proposed by Beck [27] and used in a later work [28], exactly yields Eq. (1) for $\psi(t)$.

We make the assumption of being able to generate time series with no computer time and computer memory limitation. Of course, this is an ideal condition, and in practice we shall have to deal with the numerical limits of the mathematical recipe that we adopt here to understand modulation. The reader might imagine that we have a box with infinitely many labeled balls. The label of any ball is a given number $\lambda$. There are many balls with the same $\lambda$, so as to fit the probability density of Eq. (18). We randomly draw the balls from the box and after reading the label we place the ball back in the box. Of course, this procedure implies that we are working with discrete rather than continuous numbers. However, we make the assumption that it is possible to freely increase the ball number so as to come arbitrarily close to the continuous probability density of Eq. (18).

After creating the sequence $\left\{\lambda_{j}\right\}$, we create the sequence $\left\{\tau_{i}\right\}$ with the following protocol. For any number $\lambda_{j}$, the reader must imagine that we have available a box with another set of infinitely many balls. Each ball is labeled with a number $\tau$, and in this case the distribution density is given by $\psi(\tau)=\lambda_{j} \exp \left(-\lambda_{j} \tau\right)$. To realize modulation, we adopt the following two different prescriptions:

Prescription N. 1 We create the sequence $\left\{\tau_{i}\right\} \equiv\left\{\tau_{1}^{\left(\lambda_{1}\right)}, \tau_{2}^{\left(\lambda_{1}\right)}, \ldots, \tau_{N_{d}}^{\left(\lambda_{1}\right)}, \tau_{1}^{\left(\lambda_{2}\right)}, \tau_{2}^{\left(\lambda_{2}\right)}, \ldots, \tau_{N_{d}}^{\left(\lambda_{2}\right)}, \ldots\right\} . \quad$ This means that from each box corresponding to a given $\lambda_{j}$ we draw the fixed number $N_{d}$ of waiting times. The crucial event corresponds to the choice of a new $\lambda$, and the time between the $j$ th and the $j+1$-th crucial event is $\tau_{1}^{\left(\lambda_{j}\right)}+\tau_{2}^{\left(\lambda_{j}\right)}+\cdots+\tau_{N_{d}}^{\left(\lambda_{j}\right)}$.

Prescription N. 2. The main purpose of this prescription is to create extended time intervals, with the same time duration $T_{d}$, where the sojourn times are drawn from an exponential distribution with the same parameter $\lambda$. In this case the time distance between two consecutive crucial events is fixed and equal to $T_{d}$. To realize this purpose, we work with the sequence $\left\{\tau_{i}\right\}$ $\equiv\left\{\tau_{1}^{\left(\lambda_{1}\right)}, \tau_{2}^{\left(\lambda_{1}\right)}, \ldots, \tau_{M_{1}}^{\left(\lambda_{1}\right)}, \tau_{1}^{\left(\lambda_{2}\right)}, \tau_{2}^{\left(\lambda_{2}\right)}, \ldots, \tau_{M_{2}}^{\left(\lambda_{2}\right)}, \ldots\right\}$. The times $\tau_{M_{j}}^{\left(\lambda_{j}\right)}$ are defined in such a way that $\tau_{1}^{\left(\lambda_{j}\right)}+\tau_{2}^{\left(\lambda_{j}\right)}+\cdots+\tau_{M_{j-1}}^{\left(\lambda_{j-1}\right)}$ $<T_{d}$ and $\tau_{1}^{\left(\lambda_{j}\right)}+\tau_{2}^{\left(\lambda_{j}\right)}+\cdots+\tau_{M_{j}}^{\left(\lambda_{j}\right)} \geqslant T_{d}$. As we see, the condition $\tau_{1}^{\left(\lambda_{j}\right)}+\tau_{2}^{\left(\lambda_{j}\right)}+\cdots+\tau_{M_{j}}^{\left(\lambda_{j}\right)}=T_{d}$ is not exactly realized. However, the consequences of this error are expected to become irrelevant in the limiting case of infinitely large $T_{d}$ values. We shall limit our discussion to the case of very large $T_{d}$ values, where this condition is realized with a very good approximation. This corresponds to the case of very slow modulation.

Notice that according to the arguments of Cohen [26] for modulation to be identified with superstatistics, it is necessary to make either $N_{d}$ or $T_{d}$ very large, virtually infinite. This serves the purpose of allowing the system to reach the "local form" (i.e., within a time window with the same value of $\lambda$ ) of canonical equilibrium. In the case of this paper the condition of canonical equilibrium means a form of ordinary diffusion with ordinary scaling. As described in Sec. I, after generating the time series $\left\{\tau_{i}\right\}$ with either prescription N. 1 or prescription N. 2, we convert this time series into a fluctuation $\xi(t)$, and then we use this fluctuation to generate a diffusion process. The local form of diffusion process is ordinary, as the local form of Cohen's equilibrium is canonical. The emergence of nonordinary diffusion is caused by the fact that from time to time a transition from a given exponential time scale to another occurs.

Both prescriptions yield for $\psi(t)$ the nonexponential form of Eq. (1). In this paper, as pointed out in Sec. II, we consider the condition $\mu>2$, which makes finite the mean value of $\tau$, given by Eq. (13). The corresponding renewal process is ergodic, and the correlation function of the fluctuation $\xi(t)$, $\left\langle\xi\left(t_{1}\right) \xi\left(t_{2}\right)\right\rangle$, with $t_{2}>t_{1}$, can be evaluated by adopting the time average,

$$
\left\langle\xi\left(t_{1}\right) \xi\left(t_{2}\right)\right\rangle=\lim _{T \rightarrow \infty} \frac{\int_{0}^{T} d t \xi(t) \xi\left(t+t_{2}-t_{1}\right)}{T} .
$$

Using renewal theory [29] we find for the normalized correlation function $\Phi_{\xi}(t)$ defined by

$$
\Phi_{\xi}(\tau) \equiv \frac{1}{W^{2}}\langle\xi(t) \xi(t+\tau)\rangle
$$

the following analytical expression

$$
\Phi_{\xi}(\tau)=\frac{1}{\langle\tau\rangle} \int_{\tau}^{\infty} d \tau(t-\tau) \psi(\tau),
$$

which yields

$$
\frac{d^{2}}{d t^{2}} \Phi_{\xi}(\tau)=\frac{\psi(\tau)}{\langle\tau\rangle}
$$

It is important to point out, with the authors of Ref. [15], that the existence of the stationary correlation function does not conflict with the property of renewal aging. In this case, however, the system evolves towards the equilibrium condition, which is compatible with the condition $\mu>2$, albeit the transition from the out of equilibrium to equilibrium is very slow if $\mu<3$. The stationary correlation function is a property of this equilibrium condition.

On the other hand, the distribution density $\Pi(\lambda)$ is an equilibrium distribution allowing us to evaluate the correlation function through the prescription of Eq. (19) even if we realize the inverse power law distribution of Eq. (1) through modulation rather than by means of the renewal prescription. We note that with prescription N. 1 the time spent by the 
system with a given $\lambda$ is inversely proportional to $\lambda$, whereas prescription N. 2 makes it independent of $\lambda$. Thus, the use of the prescription of Eq. (19) yields for the stationary correlation function, with prescription N. 1 and prescription N. 2 the expressions

$$
\Phi_{\xi}(\tau)=\frac{\int d \lambda \frac{\Pi(\lambda)}{\lambda} \exp (-\lambda \tau)}{\int d \lambda \frac{\Pi(\lambda)}{\lambda}}
$$

and

$$
\Phi_{\xi}(\tau)=\int d \lambda \Pi(\lambda) \exp (-\lambda \tau),
$$

respectively.

As noted by the authors of [28], in the case $2<\mu<3$, the adoption of prescription N. 1, and, consequently, of Eq. (23), makes $\psi(t)$ proportional to the second time derivative of $\Phi_{\xi}(t)$. It is straightforward to realize this fact by comparing Eq. (23) to Eq. (17). As a consequence of this property, we see that prescription N. 1 makes the renewal and the modulation approach share the same correlation function as well as the same waiting time distribution of Eq. (1). The analytical form of this correlation function is

$$
\Phi_{\xi}(t)=\left(\frac{T}{T+t}\right)^{\mu-2},
$$

which is not integrable. With prescription N. 2, on the contrary, the waiting time distribution $\psi(t)$ is proportional to the first rather than to the second time derivative of $\Phi_{\xi}(t)$. Thus, in the same case $2<\mu<3$ the correlation function becomes

$$
\Phi_{\xi}(t)=\left(\frac{T}{T+t}\right)^{\mu-1},
$$

and consequently it is integrable. This allows us to conclude that prescription N. 2 yields the ordinary scaling $\delta=0.5$. In fact, by integrating Eq. (2), squaring $x(t)$, averaging on the Gibbs ensemble, and exploiting the stationary nature of the correlation function $\left\langle\xi\left(t_{1}\right) \xi\left(t_{2}\right)\right\rangle=\left\langle\xi(0) \xi\left(t_{2}-t_{1}\right)\right\rangle$, we obtain [30]

$$
\left\langle x^{2}(t)\right\rangle=2 W^{2} \int_{0}^{t} d t^{\prime} \int_{0}^{t^{\prime}} d t^{\prime \prime} \Phi_{\xi}\left(t^{\prime \prime}\right)
$$

under the condition that all the walkers move from $x=0$ at $t=0$. By using the analytical expression of Eq. (26), we find

$$
\left\langle x^{2}(t)\right\rangle=2 W^{2} T^{2} t+\frac{2 W^{2} T^{3-\mu}}{(3-\mu)(\mu-2)}-\frac{2 W^{2}(T+t)^{3-\mu}}{(3-\mu)(\mu-2)} .
$$

This means that, although slowly, the second moment tends to increase linearly with time for $t \rightarrow \infty$. Thus, as earlier stated, prescription N. 2 leads to the scaling $\delta=0.5$. We invite the reader to keep in mind this conclusion. In fact, in Sec. V we shall reach the same conclusion by using the concept of crucial event illustrated in Sec. I.

\section{AGING EFFECTS IN RENEWAL AND MODULATION THEORIES}

The phenomenon of aging has been known for a long time as a property of spin glasses and polymers [31]. For a review on aging associated to critical phenomena we can refer the reader to the recent work of Ref. [32]. The most popular manifestation of aging is given by two time correlation functions, depending on two times $t_{1}$ and $t_{2}>t_{1}$ not only through their difference. The decay as a function of $t_{2}-t_{1}$ becomes slower for larger $t_{1}$. Aging is thought to be determined by the fact that the system under study is out of equilibrium and that the regression to equilibrium involves times larger than the observation time [32].

In this paper we consider a specific form of aging, called renewal aging, which is discussed in details in many recent papers, a sample of which is given by Refs. [7-9]. The dynamic model illustrated in Sec. II is renewal and can be used to produce this form of aging, which is revealed by the dependence of $\psi(t)$ on the observation time. In fact, the distribution density $\psi(t)$ has the following meaning: $\psi(t) d t$ is the probability that a laminar region beginning at $t=0$ ends in the small interval $[t, t+d t]$. Let us imagine that we have at our disposal a Gibbs ensemble of time series $\left\{t_{i}\right\}$. We use these time series to build up a Gibbs ensemble of symbolic sequences, with the symbol 1 when an event occurs, and the symbol 0 , when there are no events. This is equivalent to filling the laminar regions with 0's, and to assigning the symbol 1 to the border between two consecutive laminar regions. All these sequences are prepared in such a way that at $t=0$ an event occurs, so that for each of them, at $t=0$ we find the symbol 1 . To produce the histogram determining $\psi(t)$ we have to observe the time at which the first laminar region of each sequence of the sample ends. This is equivalent to making preparation and observation at the same time.

What about the case when observation is delayed with respect to preparation? Let us imagine now that preparation is made at time $t=-t_{a}<0$ and observation begins at time $t=0$. The first measured waiting time is denoted by $\tau_{1}$. The first waiting time, at variance with the observation of the successive waiting times, does not necessarily correspond to the total time duration of a laminar region. In fact, the first laminar region could have started at $t=-t_{a}$, or at an unknown later time $t=-t_{u}$, earlier, though, than the time $t=0$ at which observation begins. Thus, the real length of the laminar region corresponding to the first observed waiting time is given by $\tau_{1}+t_{u}$. The resulting histogram records time lengths that are generally smaller than those corresponding to preparing and observing the system at the same time. Nevertheless, in the case when the waiting time distribution is exponential, both long- and short-time lengths are reduced by the same percent. Thus, turning the histogram into a normalized waiting distribution density has the effect of recovering the same exponential form. A renewal exponential process does not age. In the nonexponential case delaying the observation process has the effect of producing a percent cut of the shorttime laminar regions larger than that of the long-time laminar regions. As a consequence, normalizing the histogram, so as to produce the aging waiting time distribution $\psi_{t_{a}}$, has the 
effect of reducing the weight of the short-duration laminar regions and of enhancing that of the long-duration laminar regions.

The dynamic model of Sec. II is useful to understand renewal aging. Making observation and preparation at the same time is equivalent to drawing the numbers $y_{0}$ from a uniform distribution on the interval $I$, thereby yielding by means of the nonlinear transformation of Eq. (9) the inverse power law distribution of Eq. (1). The postponement of the observation process corresponds to determining $\psi(t)$ through Eq. (10) again, with $p_{0}\left(y_{0}\right)$ replaced by the distribution $p\left(t_{a}, y_{0}\right)$. This yields the aging waiting time distribution $\psi_{t_{a}}(t)$, which depends on $t_{a}$ for the following physical reasons. The dynamic system of Sec. II is prepared in the uniform distribution $p_{0}\left(y_{0}\right)$ and it is let free to evolve in time, under the influence of the prescription of Eq. (5) and of the back-injection process. Delaying the observation by the quantity $t_{a}$ has the effect of selecting with a larger probability the smaller values of $y_{0}$. In fact $p\left(t_{a}, y_{0}\right)>p\left(t_{a}, y_{0}^{\prime}\right)$ if $y_{0}<y_{0}^{\prime}$. The experimental observation, yielding $\psi_{t_{a}}(t)$ allows us to establish at which time the system was prepared. This is a form of long-standing memory that yet is compatible with the fact that the laminar regions showing up in the future do not have any memory of those that occurred in the past.

In this section, we illustrate, with the help of a numerical example, how aging effects are deeply related to the renewal character of the process, whereas aging is annihilated by the slow modulation. Monte Carlo simulations of a renewal process with inverse-power-law distribution of waiting times, Eq. (1), are performed using the approach proposed in Sec. II, i.e., we generate a sequence $\left\{\tau_{i}\right\}$ by using Eq. (9).

As far as modulation theory is concerned, the numerical treatment is a little bit more delicate. The first step in the Monte Carlo simulation consists in generating a sequence of random numbers $\lambda$ in agreement with the $\Gamma$ distribution $\Pi(\lambda)$ given in Eq. (18). An approach similar to the one used in Sec. II cannot be applied, because there is no simple analytical expression for the cumulative function related to $\Gamma$ distributions. As a consequence, it is not possible to obtain a simple explicit expression for the function relating the random number $\lambda$ to a uniform random number in the interval $I=[0,1]$. For this reason, we decided to use the rejection method suggested by Ref. [33]. This method is based on the use of a majorant function $f(\lambda)$, i.e., a function that, for each point, takes values slightly greater than the corresponding values of the $\Gamma$ distribution. The general idea is to draw random points in the plane, uniformly distributed under the graph of $f(\lambda)$. Then, the points under the graph of the $\Gamma$ distribution are accepted and the others rejected. The random points are drawn as follows. First of all, we draw a random number $\lambda$ distributed as the probability distribution $f(\lambda) / A$, where $A>1$ is the area under the graph of $f(\lambda)$. This is easily done if $f(\lambda)$ is chosen with a simple analytical expression, in order to apply the method of Sec. II. Then, given $\lambda$, a uniform random number $\zeta$ is drawn in the interval $[0, f(\lambda)]$. The coordinates $(\lambda, \zeta)$ define the random point. Finally, if $\zeta$ is smaller than the corresponding value $\Pi(\lambda)$, then $\lambda$ is accepted, otherwise it is rejected. Without loss of generality we fix $T=1$ and we chose the following majorant function:

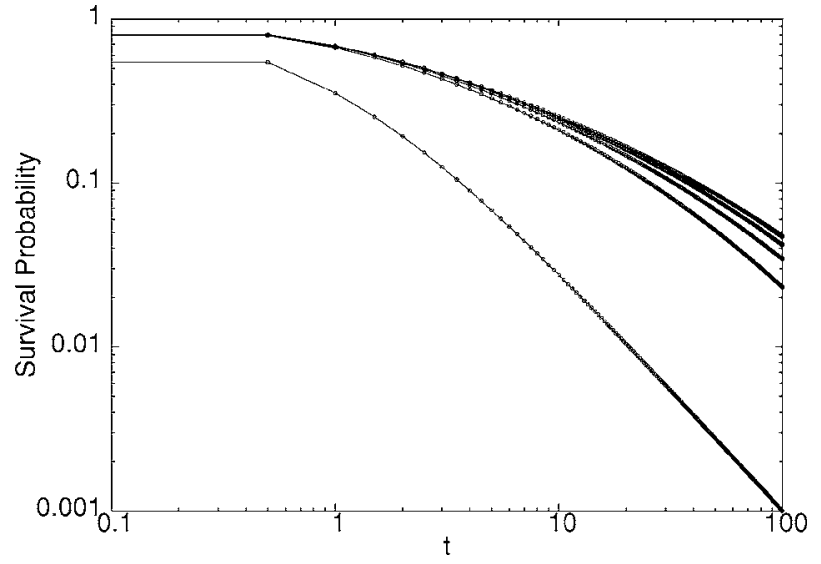

FIG. 1. Comparison between the function $\Psi_{t_{a}}$ of a renewal process (continuous lines) and the $\Psi_{t_{a}}$ of a modulation process with $N_{d}=1$ (dots) at different values of $t_{a}=0,50,100,150,200$ (from the lower to the upper curves).

$$
f(\lambda)= \begin{cases}\gamma e^{-\lambda} & \text { if } \lambda \leqslant 1 ; \\ \frac{\eta}{\lambda^{2}} & \text { if } \lambda>1 .\end{cases}
$$

According to Ref. [33], in order to obtain a certain number of accepted numbers, the rejection method requires a greater amount of random drawings, depending on the choice of the majorant function. We found that, given the majorant function described by Eq. (29), with $\eta=1.7$ and $\gamma=\eta e$ ( $e$ is the Neper's number), the total number of drawings is about the double of the accepted drawings. Furthermore, we compared the histograms computed from the sequence of simulated random numbers with the relative probability density distribution $\Pi(\lambda)$ given in Eq. (18), with excellent agreement. Following Prescription N. 1, for each number $\lambda$ we have to draw $N_{d}$ numbers from the exponential distribution described in Eq. (15), representing a sequence of waiting times. This is easily obtained with the same standard method described in Sec. II. In this Section we use this approach to generate trajectories, i.e., artificial sequences of random waiting times. We compare the results for the aging analysis of trajectories characterized by the same exponent $\mu$ of the powerlaw, but generated from the renewal process and from modulation processes with different $N_{d}$.

We perform the aging analysis in the following way. Given the sequence of waiting times and an aging time $t_{a}$, we compute the truncated waiting times, i.e., the difference between each waiting time and $t_{a}$. When the waiting time is shorter than $t_{a}$, then we take the successive waiting times until their sum exceeds $t_{a}$. Then the truncated waiting time is defined as the difference between this sum and $t_{a}$. In this way, we obtain a sequence of truncated waiting times characterized by some aged probability density distribution $\psi_{t_{a}}(\tau)$. Aging is revealed if this distribution changes with $t_{a}$. Note that the modulation process resting on Prescription N. 1 , for $N_{d}=1$ is expected to coincide with the prediction of renewal theory. To confirm this important property by means of numerical calculation, in Fig. 1 we make a comparison between the renewal and the modulation process, with 


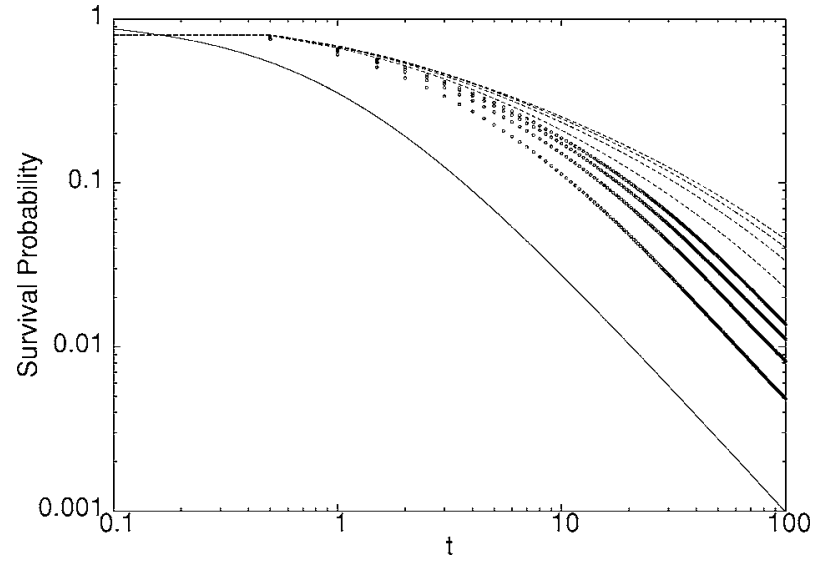

FIG. 2. Comparison between the function $\Psi_{t_{a}}$ of a renewal process (dashed lines) and the function $\Psi_{t_{a}}$ of a modulation process with $N_{d}=10$ (dots), at different values of $t_{a}=50,100,150,200$ (from the lower to the upper curves). The lowest curve represents the brand new function $\Psi(t)$.

$N_{d}=1$. For graphical reasons, rather than measuring the function $\psi_{t}$, we evaluate the aged survival probability $\Psi_{t a}$, which is related to $\psi_{t_{a}}$ by the following relation:

$$
\Psi_{t_{a}}(\tau)=\int_{\tau}^{\infty} \psi_{t_{a}}\left(\tau^{\prime}\right) d \tau^{\prime}=1-\int_{0}^{\tau} \psi_{t_{a}}\left(\tau^{\prime}\right) d \tau^{\prime},
$$

conveying therefore the same information of $\psi_{t_{a}}$.

The results of the numerical experiments are illustrated by Figs. 1-4. All these four figures illustrate the aging effect on both the renewal (continuous lines) and the modulation model (dotted lines). Figure 1 refers to the case $N_{d}=1$. In this case, the choice of a given $\lambda$ determines only one waiting time. As a consequence, any laminar region does not have anything in common with the earlier laminar regions, and modulation is indistinguishable from renewal, as shown by Fig. 1. On the contrary, Fig. 2 shows that it is enough to set $N_{d}=10$ to make the dotted lines significantly depart from the dashed lines. We see that the dotted lines indicate a faster decay than the dashed lines, which implies that with modulation the aging effect is significantly reduced already at

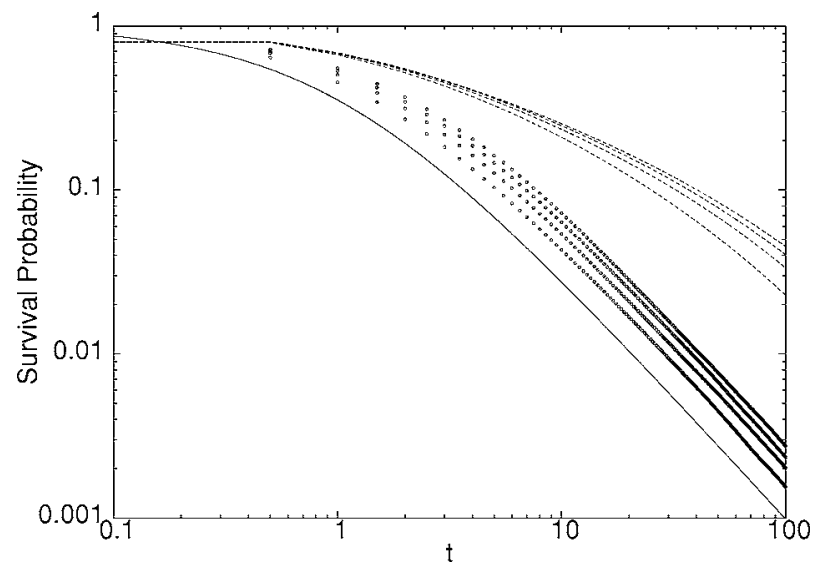

FIG. 3. The same as Fig. 2, but with $N_{d}=100$.

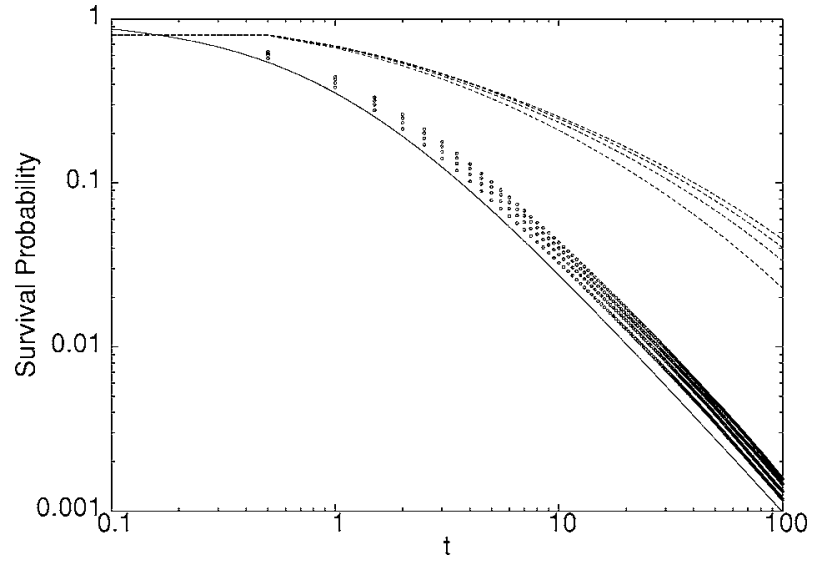

FIG. 4. The same as Fig. 2, but with $N_{d}=500$.

$N_{d}=10$. With a further increase of $N_{d}$ (see Figs. 3 and 4) this effect becomes more pronounced. We see, in fact, that the dotted lines tend to overlap with the brand new survival probability. We conclude that for $N_{d} \rightarrow \infty$ the aging effect is annihilated. We thus conclude that superstatistics, as a form of infinitely slow modulation, yields no aging, in accordance with the prediction of Ref. [10].

\section{DIFFUSION}

We address the problem of diffusion generated by modulation using two distinct procedures: Prescriptions N. 1 and N. 2 of Sec. II. We shall use the approach of the ContinuousTime Random Walk (CTRW) [34] to derive the asymptotic scaling of the process. The problem of diffusion with renewal has been the object of earlier work [35] to which we refer the interested reader to appreciate the difference between renewal and modulation on this specific issue. Here we limit ourselves to remarking that the renewal diffusion realizes the scaling condition of Eq. (3) with $\delta=1 /(\mu-1)$ and $F$ given by a symmetric Lévy $(\mu-1)$-stable function. These are properties of the central part of the PDF, which is truncated by ballistic peaks. As a consequence, the condition of Eq. (3) is not exactly fulfilled and the resulting diffusion process is multi-scaling [35].

\section{A. Continuous time random walk: Fixed number of drawings}

We remind the reader that in Sec. I we have mentioned the important role of the abrupt changes of exponential time scale. We have decided to denote these abrupt changes of exponential time scale as crucial events. More precisely, the crucial event corresponds to drawing from a given exponential distribution the time length of a laminar region for the first time. Let us assume that the drawing of the first waiting time from a given exponential distribution and the drawing of $\lambda$ from $\Pi(\lambda)$ occur at the same time. Thus, we identify the crucial events with the drawings of $\lambda$ 's from the distribution $\Pi(\lambda)$.

We plan to proceed as in the well known CTRW theory [34] extended by Zumofen and Klafter [13] to the case where the particle moves with constant velocity, in either the posi- 
tive or negative direction, in between two consecutive renewal events. With this theory it is essential to define and use the properties $\psi(t, x), \psi_{n}(x, t)$, and $\Psi(t, x)$. The first is the probability density for the walker to move a distance $x$ in time $t$ in a single motion event, the second is the probability density that the walker moves a distance $x$ as a result of $n$ events, the last of which makes the walker arrive at $x$ exactly at time $t$. Finally, the last property is the probability for the walker to move a distance $x$ with no event occurring up to time $t$. We plan to build up the corresponding properties $P(x, t), P^{(n)}(t)$, and $N(x, t)$, where the CTRW events are replaced by the crucial events. It is evident that when $N_{d}=1$ this generalized theory coincides with the ordinary CTRW $[13,34]$. With these generalized properties we express the probability density $p(x, t)$ for the walker to be at position $x$ at time $t$ as follows

$p(x, t)=\sum_{n=1}^{\infty} \int_{0}^{\infty} d t^{\prime} \int_{-\infty}^{+\infty} d x^{\prime} P^{(n)}\left(x^{\prime}, t^{\prime}\right) N\left(x-x^{\prime}, t-t^{\prime}\right)+N(x, t)$.

To solve the intriguing problem of determining the scaling produced by prescription N. 1, we shall use the FourierLaplace transform of Eq. (31), which reads

$$
\hat{p}(k, u)=\frac{1}{1-\hat{P}(k, u)} \hat{N}(k, u) .
$$

Note that to get this result we have to use the property

$$
\hat{P}^{n}(k, u)=(\hat{P}(k, u))^{n},
$$

which is a consequence of the fact that the occurrence of different crucial events produce uncorrelated motional effects on the random walker.

We have to explain now how to build up $P(x, t)$, $P^{(n)}(x, t)$, and $N(x, t)$, by keeping in mind that according to prescription N. 1, we have to draw $N_{d}$ waiting times from the same exponential waiting time distribution

$$
\psi_{\lambda}(\tau)=\lambda \exp (-\lambda \tau)
$$

Let us define first the function $\psi^{(i)}(x, t)$. This is the probability density of moving by the quantity $x$ in time $t$, with $i$ time drawings from the same Poisson distribution. This important function reads

$$
\psi^{(i)}(x, t)=\int_{0}^{\infty} d \lambda \psi_{\lambda}^{(i)}(x, t) \Pi(\lambda),
$$

where $\psi_{\lambda}^{(i)}(x, t)$ denotes the probability density of moving by $x$ in time $t$ as a result of drawing $i$ numbers from a given Poisson distribution, thereby corresponding to the same value of the parameter $\lambda$. The functions $\psi_{\lambda}^{(i)}(x, t)$ obey the following recursion relation:

$$
\psi_{\lambda}^{(i)}(x, t)=\int_{0}^{t} d t^{\prime} \int_{-\infty}^{+\infty} d x^{\prime} \psi_{\lambda}^{(i-1)}\left(x^{\prime}, t^{\prime}\right) \psi_{\lambda}^{(1)}\left(x-x^{\prime}, t-t^{\prime}\right),
$$

with

$$
\psi_{\lambda}^{(1)}(x, t) \equiv \lambda \exp (-\lambda t) \frac{1}{2}[\delta(x-W t)+\delta(x+W t)] .
$$

We remind the reader that the symbol $W$ and $-W$ are values assigned to the laminar regions by means of a fair coin tossing prescription (see Sec. I). This explains the two deltas of Dirac, indicating that the particle with the same probability, $1 / 2$, can travel with constant velocity in both the positive and negative direction. In conclusion, the physical meaning of the function $\psi^{(i)}(x, t)$ of (35) corresponds to adopting the following procedure. We draw a given $\lambda$ from a set of many $\lambda$ values with the probability density $\Pi(\lambda)$. Then, we draw $i$ waiting times $\tau$ from the corresponding distribution density $\psi_{\lambda}(\tau)=\lambda \exp (-\lambda \tau)$. For very large values of $i$, the $\lambda$ subsequence, i.e., the portion of the time series $\left\{t_{i}\right\}$ corresponding to $\lambda$, has a time length equal to $i / \lambda$. Let us assume that the $\lambda$ subsequences are large enough as to produce diffusion and to move the particle by a fluctuating quantity $x$, which can be evaluated using the ordinary diffusion equation.

We are now in a position to express the quantity $P(x, t) \equiv P^{(1)}(x, t)$ as follows

$$
P^{(1)}(x, t)=\psi^{\left(N_{d}\right)}(x, t) .
$$

In other words, the transition by the quantity $x$ in time $t$ generated by a single crucial event is derived from Eq. (36) by setting $i=N_{d}$. As far as $N(x, t)$ is concerned, we have

$$
N(x, t)=\int_{0}^{+\infty} d \lambda \Pi(\lambda) N_{\lambda}(x, t)
$$

and

$$
N_{\lambda}(x, t)=\sum_{i=1}^{N_{d}} \int_{0}^{t} d t^{\prime} \int_{-\infty}^{+\infty} d x^{\prime} \psi_{\lambda}^{(i-1)}\left(x^{\prime}, t^{\prime}\right) \Psi_{\lambda}\left(x-x^{\prime}, t-t^{\prime}\right),
$$

where with $\psi_{\lambda}^{(0)}(x, t)$ we denote the initial condition $\delta(x) \delta(t)$. In (40) there are no crucial events involved because the maximum numbers of time drawings is $N_{d}-1$, not enough to generate a new crucial event, if we start at time $t=0$ with a crucial event. As a result of this partial number of drawings the particle reaches position $x^{\prime}$ in time $t^{\prime}$. The remainder portion of space $x-x^{\prime}$ in the remainder portion of time $t-t^{\prime}$ is traveled with no further time drawing, according to the prescription

$$
\Psi_{\lambda}(x, t)=\frac{[\delta(x+W t)+\delta(x-W t)]}{2} \int_{t}^{+\infty} d t^{\prime} e^{-\lambda t^{\prime}} .
$$

At this stage we are properly equipped to evaluate $\hat{p}(k, u)$ of Eq. (32). This requires the evaluation of the FourierLaplace transform of $P(x, t)$ and $N(x, t)$, and, consequently, according to the earlier equations, the Fourier-Laplace transform of $\psi^{(i)}(x, t), \Psi_{\lambda}(x, t)$, and $P(x, t)$. Using the convolution theorem we have:

$$
\hat{\psi}^{(i)}(k, u)=\int_{0}^{+\infty} d \lambda \Pi(\lambda)\left[\frac{\lambda(u+\lambda)}{(u+\lambda)^{2}+k^{2} W^{2}}\right]^{i}
$$

and 


$$
\hat{\Psi}_{\lambda}(k, u)=\frac{(u+\lambda)}{(u+\lambda)^{2}+k^{2} W^{2}} .
$$

From Eqs. (42) and (38) we derive immediately

$$
\hat{P}(k, u)=\int_{0}^{+\infty} d \lambda \Pi(\lambda)\left[\frac{\lambda(u+\lambda)}{(u+\lambda)^{2}+k^{2} W^{2}}\right]^{N_{d}} .
$$

To evaluate the scaling of the diffusion process we have to study the asymptotic limit, $u \rightarrow 0$ and $k \rightarrow 0$. In the asymptotic limit the numerator on the right-hand side of Eq. (32) tends to constant value

$$
\hat{N}(0,0)=N_{d} \int d \lambda \frac{\Pi(\lambda)}{\lambda} .
$$

This means that the scaling emerges from the denominator of the term on the right hand side of Eq. (32). It is easy to deal with this scaling generating term if we go to the asymptotic limit first and then we make the average on $\lambda$. By making the asymptotic expansion of Eq. (44) and using Eq. (45) we arrive at the final result

$$
\hat{p}(k, u)=\frac{1}{u+k^{2} W^{2}\left\langle\frac{1}{\lambda}\right\rangle_{\lambda}}
$$

where the angle brackets with the subscript $\lambda$ indicate an average over the weight $\Pi(\lambda) / \lambda$ as in Eq. (23). In other words,

$$
\left\langle\frac{1}{\lambda}\right\rangle_{\lambda}=\frac{\int_{0}^{\infty} d \lambda \frac{\Pi(\lambda)}{\lambda^{2}}}{\int_{0}^{\infty} d \lambda \frac{\Pi(\lambda)}{\lambda}}
$$

This result, stemming from going to the asymptotic limit before averaging, cannot be correct because it would produce a diverging diffusion coefficient, when $\mu<3$. It signals anyway that there must be an extended time region where the diffusion process is characterized by the scaling

$$
\delta=0.5
$$

This is the condition of an ensemble of Poisson processes, each of them characterized by a fixed value of $\lambda$, selected from the distribution $\Pi(\lambda) / \lambda$ and then kept fixed forever. In this condition, aging would be totally annihilated, in accordance with Ref. [10].

\section{B. The waiting time distribution of time distances between two consecutive crucial events}

As stated in Sec. I, in this paper we aim at studying the case where modulation is slow, but not infinitely slow. Thus, we have to go beyond the assumption made in the earlier subsection. We use Eq. (36) and with some algebra, in addition to Eq. (37), we find

$$
\begin{aligned}
\psi_{\lambda}^{(2)}(x, t)= & \frac{\lambda^{2} t \exp (-\lambda t)}{4} \\
& \times\left\{[\delta(x-W t)+\delta(x+W t)]+\frac{\theta(W t-|x|)}{W t}\right\}
\end{aligned}
$$

and

$$
\begin{aligned}
\psi_{\lambda}^{(3)}(x, t)= & \frac{\lambda^{3} t^{2} \exp (-\lambda t)}{16} \\
& \times\left\{[\delta(x-W t)+\delta(x+W t)]+3 \frac{\theta(W t-|x|)}{W t}\right\},
\end{aligned}
$$

where $\theta$ denotes the step Heaviside function. We see that the functions $\psi_{\lambda}^{(i)}(x, t)$ with $i>1$ are characterized by a nonvanishing contribution between the two deltas of Dirac. This contribution makes it difficult to move from $\psi_{\lambda}^{(i)}(x, t)$ to $\psi^{(i)}(x, t)$, according to the prescription of Eq. (35), but in the case $i=1$, yielding

$$
\psi^{(1)}(x, t)=\frac{1}{2}[\delta(x-W t)+\delta(x+W t)] \psi(t)
$$

where $\psi(t)$, thanks to the average over $\lambda$, gets the same analytical form as Eq. (1).

This suggests to make a first attempt at evaluating the scaling of this diffusion process by neglecting the nonballistic contribution to $\psi_{\lambda}^{(i)}(x, t)$. We denote these approximated expressions for $\psi_{\lambda}^{(i)}(x, t)$ with the symbol $\Lambda_{\lambda}^{(i)}(x, t)$. It is possible to find a general and exact expression for $\Lambda_{\lambda}^{(i)}(x, t)$, which reads

$$
\Lambda_{\lambda}^{(i)}(x, t)=\frac{1}{2^{i}}[\delta(x-W t)+\delta(x+W t)] \frac{\lambda^{i} t^{i-1}}{i-1 !} \exp (-\lambda t) .
$$

In this expression we identify three factors with a specific meaning. The factor $2^{-i}$ is a signature of the fact that for each ballistic contribution we tossed the coin $i$ times, always getting the same result. The second term $[\delta(x-W t)+\delta(x+W t)]$ means that we are considering only the ballistic motion. Finally in the third term, $\lambda^{i} t^{i-1} \exp (-\lambda t) /(i-1)$ ! one can easily recognize the Poisson distribution of obtaining the $i$ th success at the $t$ th trial, in a urn model [36]. This indicates that the elapsed time is generated by $i$ distinct drawings from the same Poisson distribution, with the same $\lambda$.

The expression of Eq. (52) makes it easy to evaluate the approximated expression corresponding to $\psi^{(i)}$, denoted by $\Lambda^{(i)}$. This is derived from Eq. (52) by making the proper average on $\lambda$ with the statistical weight proportional to $\Pi(\lambda)$. After some algebra we obtain 


$$
\begin{aligned}
\Lambda^{(i)}(x, t)= & \frac{1}{(i-1) ! 2^{i}}[\delta(x-W t)+\delta(x+W t)] \\
& \times\left[\frac{\partial^{i-1}}{\partial \epsilon^{i-1}} \int_{0}^{\infty} d \lambda \Pi(\lambda) \lambda \exp (\lambda \epsilon t)\right]_{\epsilon=-1},
\end{aligned}
$$

which yields

$$
\begin{aligned}
\Lambda^{(i)}(x, t)= & {[\delta(x-W t)+\delta(x+W t)] } \\
& \times \frac{(\mu-1) \mu \cdots(\mu+i-2) T^{\mu-1} t^{i-1}}{(i-1) ! 2^{i}(t+T)^{\mu+i-1}} .
\end{aligned}
$$

At this stage, we are ready to find the waiting time distribution of the times of sojourn between two consecutive crucial events, denoted by us as $\psi_{\text {crucial }}(t)$. This distribution density is derived from Eq. (54) by setting $i=N_{d}$ and by integrating over $x$. This procedure yields

$$
\begin{aligned}
\psi_{\text {crucial }}(t)= & \frac{1}{\left(N_{d}-1\right) ! 2^{N_{d}-1}}(\mu-1) \mu \cdots\left(\mu+N_{d}-2\right) \\
& \times \frac{T^{\mu-1} t^{N_{d^{-1}}}}{(t+T)^{\mu+N_{d^{-1}}}} .
\end{aligned}
$$

We see that this distribution density vanishes at $t=0$, and it becomes an inverse power law at very large times. To estimate the time necessary for this distribution to become indistinguishable from an inverse power law, we evaluate the time at which it reaches a maximum. The larger this time, the larger the time necessary for this waiting time distribution to reach the asymptotic condition. This time turns out to be

$$
t=t_{\text {max }} \equiv \frac{\left(N_{d}-1\right) T}{\mu},
$$

namely, for large values of $N_{d}$ it is proportional to the number of time drawings from the same exponential waiting time distribution. Thus, increasing $N_{d}$ has the effect of postponing the transition from the microscopic to the asymptotic regime. When the asymptotic regime is eventually reached, we see the emergence of an inverse power law with index $\mu$. Thanks to the renewal character of the crucial events, it is well known, see, for instance, $[12,13]$, that the scaling coefficient $\delta$ gets the following expression

$$
\delta=\frac{1}{\mu-1},
$$

which is identical to Eq. (4). It is important to notice that Eq. (4) is the scaling that we derive from the experimental histogram under the assumption that all the recorded events are of renewal. In the modulation case, the assumption that all the recorded events are renewal is incorrect. In the specific case of prescription N. 1, we find that the waiting time distribution $\psi_{\text {crucial }}(t)$ of Eq. (55) has the same power index of the distribution density of the time distances between two consecutive recorded events, thereby forcing diffusion to eventually reach the same scaling as the genuinely renewal model.

\section{A heuristic derivation of diffusion scaling}

Which is the effect of the nonballistic contribution? In the earlier subsection we have made the assumption of neglecting the nonballistic contribution to diffusion. Cattaneo [37] found the exact solution for a diffusion process corresponding to drawing the waiting times always from the same exponential waiting time distribution and showed that in the long-time limit the ballistic peaks can be neglected and the diffusion process becomes indistinguishable from the ordinary Gaussian diffusion. Thus now we adopt the approximation that the walker moving with the same exponential prescription in a time $t$ travels a distance $x$ with the probability density

$$
p_{\lambda}(x, t)=\frac{1}{\left(4 \pi W^{2} t / \lambda\right)^{1 / 2}} \exp \left(\frac{-x^{2} \lambda}{4 W^{2} t}\right) .
$$

This approximation allows us to define the function $M(x)$, which is the probability density for the walker to travel the distance $x$ in the time interval between two consecutive critical events. We notice that the time duration of the motional event created by the same exponential waiting time distribution with time scale $1 / \lambda$ is given by $t_{c} \approx N_{d} / \lambda$. Thus, $M(x)$ is obtained from $p_{\lambda}(x, t)$ of Eq. (58) by setting $t=t_{c}$ and averaging on all possible $\lambda$ with the statistical weight $\Pi(\lambda)$, thereby yielding

$$
\begin{aligned}
M(x)= & \frac{T^{\mu-1}}{\Gamma(\mu-1)} \times \int_{0}^{\infty} \frac{\lambda}{\left(4 \pi W^{2} N_{d}\right)^{1 / 2}} \\
& \times \exp \left(\frac{-x^{2} \lambda^{2}}{4 W^{2} N_{d}}\right) \lambda^{\mu-2} \exp (-\lambda T) d \lambda .
\end{aligned}
$$

Let us notice that the power index $\mu$ of the distribution density $\psi_{\text {crucial }}(\tau)$ of the earlier subsection meets the condition $2<\mu<3$. This means that the time distance between two consecutive crucial events, $\tau_{\text {crucial }}$, exists and has the finite value $\left\langle\tau_{\text {crucial }}\right\rangle$, whose explicit expression is not relevant for the present discussion. We limit ourselves to noticing that in the large asymptotic time limit the number of crucial events is $n=t /\left\langle\tau_{\text {crucial }}\right\rangle$, and the resulting distribution $p(x, t) \approx p(x, n)$ is determined by the generalized central limit theorem [38]. As proved in Ref. [35], the generalized central limit theorem is a reliable tool to determine the probability distribution density emerging from the nonballistic contributions. According to Ref. [38], if the second moment of $M(x)$ is finite, the system falls in the basin of attraction of Gauss diffusion and $\delta=0.5$. If, on the contrary, the second moment of $x$ is divergent, the system falls in the basin of attraction of Lévy diffusion, the diffusion process being $\alpha$-stable. This means that to recover finite moments we have to study the fractional moments $\left\langle|x|^{\alpha}\right\rangle$, with $\alpha<2$. Note that the index $\mu$ of the Lévy inverse power law is $\mu=\alpha+1$. Thus, a heuristic way to establish if the process is of Lévy kind or not, rests on the evaluation of the fractional moment $\left\langle|x|^{\alpha}\right\rangle$, with the weight $M(x)$ of Eq. (59). Then, for any $\alpha$ we must establish which is the threshold value of $\mu$. By threshold value of $\mu$ we mean the value of $\mu$, below which the fractional moment 
$\left\langle|x|^{\alpha}\right\rangle$ diverges. The scaling $\delta$ is given by $\delta=1 / \alpha=1 /(\mu-1)$, with $\mu$ being the threshold value.

In other words, we set $\alpha<2$, and we look for the minimum value of $\mu$ ensuring

$$
\int_{-\infty}^{+\infty} d x|x|^{\alpha} \int_{0}^{+\infty} d \lambda \lambda^{\mu-1} \exp \left[\frac{-(x \lambda)^{2}}{4 W^{2} N_{d}}-\lambda T\right]<\infty .
$$

We reverse the integration order and use the fact that the Gaussian moments are always finite, to get

$$
\int_{-\infty}^{+\infty} d x|x|^{\alpha} \lambda^{\alpha+1} \exp \left[\frac{-(x \lambda)^{2}}{4 W^{2} N_{d}}\right]=C .
$$

Note that $C$ is independent of of $\lambda$. Thus the condition of (61) becomes

$$
\int_{0}^{+\infty} d \lambda \lambda^{\mu-\alpha-2} \exp (-\lambda T)<\infty .
$$

Note that the only source of divergence for this integral is the limiting condition $\lambda \rightarrow 0$. In fact the exponential factor $\exp (-\lambda T)$ ensures convergence for large values of $\lambda$. Thus, to ensure the convergence of this integral we must focus on the short values of $\lambda$. We see that the integral convergence is ensured by $\mu>\alpha+1$, which means that for $\alpha$ fitting the condition $1<\alpha<2$, the values of $\mu$ satisfying the condition $2<\mu<3$ are threshold values. According to the earlier illustrated criterion, this yields for the scaling $\delta=1 / \alpha$, $\delta=1 /(\mu-1)$, thereby confirming the Lévy scaling of Eq. (57).

\section{Scaling with prescription N. 1: An exact prediction}

We have seen that the function $P(x, t)$ with the LaplaceFourier transform given by Eq. (44) determines the diffusion scaling of the process and that making the asymptotic limit $k \rightarrow 0$ and $u \rightarrow 0$ before averaging over $\lambda$ is not correct. Thus, we address this scaling issue with a different procedure that yields an exact result. We focus our attention on $\hat{p}(k, 0)$, which, according to the Lévy theory [39], should be $p(k, 0) \approx 1-$ const $k^{\alpha}$. Using (32) we get the Lévy asymptotic property provided that $\hat{P}(k, 0) \approx 1-$ const $k^{\alpha}$. Note that we have to prove that $\alpha=\mu-1$. For this purpose we focus on Eq. (44). First of all, without any loss of generality, we set $W=1$ and $T=1$; then, considering $u=0$, and substituting $y=\lambda / k$, we obtain:

$$
\hat{P}(k, 0)=\frac{k^{\mu-1}}{\Gamma(\mu-1)} \int_{0}^{\infty} y^{\mu-2} e^{-T k y}\left(\frac{y^{2}}{y^{2}+1}\right)^{N} d y .
$$

At this point, we use the binomial expansion and we get:

$$
\left(\frac{y^{2}}{y^{2}+1}\right)^{N}=\frac{y^{2 N}}{\sum_{i=0}^{N}\left(\begin{array}{c}
N \\
i
\end{array}\right) y^{2 i}}=1-\frac{\sum_{i=0}^{N-1}\left(\begin{array}{c}
N \\
i
\end{array}\right) y^{2 i}}{\sum_{i=0}^{N}\left(\begin{array}{c}
N \\
i
\end{array}\right) y^{2 i}} .
$$

Let us plug this expression into the integral, and divide it into two parts:

$$
\begin{aligned}
& \frac{k^{\mu-1}}{\Gamma(\mu-1)} \times\left[\int_{0}^{\infty} y^{\mu-2} e^{-T k y} d y\right. \\
& \left.-\int_{0}^{\infty} \frac{\sum_{i=0}^{N-1}\left(\begin{array}{c}
N \\
i
\end{array}\right) y^{2 i}}{\sum_{i=0}^{N}\left(\begin{array}{c}
N \\
i
\end{array}\right) y^{2 i}} y^{\mu-2} e^{-T k y} d y\right] .
\end{aligned}
$$

The first term gives $\Gamma(\mu-1) / k^{(\mu-1)}$ and, therefore, the unity term in the zeroth order of $\hat{P}(k, 0)$. Looking at the second term, note that the integrand does not diverge in zero, at the infinity either: the integral can be considered as a Laplace transform (in $T k$ ) of a finite quantity. So, at this first order, we obtain a constant.

In conclusion, for the integral (63) we obtain the expression 1 -const $k^{\mu-1}$, which is the same first-order expansion as that stemming from the renewal process, thus yielding (57). We think that at this stage the validity of Eq. (57) is widely proved. In spite of the approximation made to get the Lévy scaling, Eq. (57), this property is correct and is expected to show up in the time asymptotic limit. We note also that the scaling of Eq. (57) is the same as that one would predict by means of the observation of $\psi(\tau)$ of Eq. (1), supplemented by the assumption that the process is renewal. However, this is a coincidence, due to the fact that $\psi_{\text {crucial }}(t)$, determining the time distance between two consecutive crucial events, has the same power index of the distribution of Eq. (1). In the next subsection we shall see that when the power index of $\psi_{\text {crucial }}(t)$ is different from that of of $\psi(\tau)$, the diffusion scaling is determined by the former distribution.

\section{E. Continuous time random walk: Fixed time $T_{d}$ for the action of the same $\lambda$}

We have focused so far our attention on the modulation prescription N. 1. Let us consider now the modulation prescription N. 2. In the case where we keep drawing the times $\tau$ from the same $\lambda$ for a time $T_{d}$, assumed to be very large, we can apply the following argument. The probability density for the particle to travel by the quantity $x$ is given by

$$
Q(x) \equiv p\left(x, T_{d}\right)=\int_{0}^{+\infty} d \lambda \Pi(\lambda) p_{\lambda}\left(x, T_{d}\right),
$$

where $p\left(x, T_{d}\right)$ denotes the probability density of traveling by the quantity $x$, in the positive, $x>0$, or negative, $x<0$, direction, for a time $T_{d}$, throughout which the random times $\tau_{i}$ has been always drawn from the same Poisson distribution of Eq. (34), with a fixed value of $\lambda$. Note that also with prescription N. 2 the crucial events correspond to the time when a new value of $\lambda$ is drawn. As a consequence of the random choice of $\lambda$, as well as of the random choice of $\tau_{i}$, the function $Q(x)$ must be interpreted as a distribution of totally uncorrelated numbers. Therefore, as done earlier, we have to focus now our attention on the fractional moments of $Q(x)$. Notice that, with prescription N. 2, the jumps occur at regu- 
lar times, separated by the fixed distance $T_{d}$, a property that makes easier and even more rigorous our scaling evaluation.

For this prescription we have

$$
p_{\lambda}\left(x, T_{d}\right)=\frac{1}{\left(4 \pi W^{2} T_{d} / \lambda\right)^{1 / 2}} \exp \left(\frac{-x^{2} \lambda}{4 W^{2} T_{d}}\right) .
$$

Substituting (58) into Eq. (66), we obtain:

$$
p\left(x, T_{d}\right)=\frac{\int_{0}^{\infty} \lambda^{1 / 2} \exp \left(\frac{-x^{2} \lambda}{4 W^{2} T_{d}}-\lambda T\right) \lambda^{\mu-2} T^{\mu-1} d \lambda}{\left(4 \pi W^{2} T_{d}\right)^{1 / 2} \Gamma(\mu-1)} .
$$

Let us consider for simplicity $T=1$ :

$$
\begin{aligned}
p\left(x, T_{d}\right)= & \frac{1}{\Gamma(\mu-1)} \frac{1}{\left(4 \pi W^{2} T_{d}\right)^{1 / 2}} \\
& \times \int_{0}^{\infty} \exp \left[-\left(\frac{x^{2}}{4 W^{2} T_{d}}+1\right) \lambda\right] \lambda^{\mu-3 / 2} d \lambda .
\end{aligned}
$$

Now, using integral 3.381(4) of Ref. [40],

$$
\int_{0}^{\infty} x^{\nu-1} e^{-\mu x} d x=\frac{1}{\mu^{\nu}} \Gamma(\nu)
$$

with $\operatorname{Re}\{\mu\}>0$ and $\operatorname{Re}\{\nu\}>0$, we obtain

$$
Q(x)=\frac{\Gamma(\mu-1 / 2)}{\Gamma(\mu-1)} \frac{1}{\left(4 \pi W^{2} T_{d}\right)^{1 / 2}}\left(\frac{4 W^{2} T_{d}}{x^{2}+4 W^{2} T_{d}}\right)^{\mu-1 / 2} .
$$

We see that for $\mu>2$, the second moment of $Q$ is finite. Thus, in this case the standard central limit theorem applies, and consequently modulation realized with prescription N. 2 yields

$$
\delta=0.5
$$

Note that with Eq. (28) we have reached the same conclusion on the basis of the properties of the correlation function $\Phi_{\xi}(t)$. It should be now evident to the reader that this correlation function is integrable for the very same reasons why in the time asymptotic time limit we can apply the central limit theorem. Equation (28) shows that the ordinary scaling is reached after a very long transient. The time distance between two consecutive crucial events, although fixed, is very large, and this make it possible for us to explain, in terms of crucial events, why the transition process to scaling is so slow.

\section{CONCLUDING REMARKS}

Let us discuss which are the main results emerging from this paper. First of all, we found that the waiting time distribution is an ambiguous indicator of complexity. This confirms the conclusion of earlier work [41] from within a new perspective made attractive by the modulation, or superstatistics, approach to complexity [26,27]. It is remarkable that the time sequence generated according to the modulation prescription would pass the randomness test, based on the use of the correlation function method. In fact, as we have seen, the correlation enforced by modulation refers to the persistent use of the same Poisson prescription to generate waiting times that are otherwise totally uncorrelated. We have seen that the adoption of the aging experiment of Sec. IV bypasses the limits of the conventional methods of analysis, since it establishes beyond any doubt the difference between the renewal and the modulation character of a sequence of times. This is a fact of some physical importance for the physics of blinking quantum dots [42]. The work of Brockmann et al. [43] has already established aging in the intermittent fluorescence of these new materials, and the more recent work of Ref. [14] confirms that this aging is due to the renewal character of the process. Thus, we conclude, in agreement with Refs. $[10,14]$, that the dynamic process responsible for intermittent fluorescence must be built up on the basis of a renewal perspective.

The second result of this paper is that the physical realization of modulation might produce renewal events. Thus, the phenomenon of renewal aging is not totally annihilated, but rather strongly reduced. This means that, as argued in Ref. [10], aging is totally annihilated only in the limiting case of $N_{d}=\infty$, which corresponds to the case of infinitely slow modulation. A reduced, but not vanishing, aging corresponds to the enhancement of the time duration of the regime of transition from microscopic dynamics to scaling. It is evident, therefore, that crucial events are responsible for both aging and scaling.

Finally, it is worth to make some remarks on the concept of crucial event. In this paper we have defined the crucial events as the abrupt changes of exponential time scales. However, this definition of crucial event is valid only in the specific case illustrated in this paper. The results of the earlier work of Ref. [41] lead us to conclude that a more general definition should be used. The authors of [41] studied the heartbeat processes and monitored the heart beat sojourn times in a given fixed frequency state. This approach generates the time series $\left\{t_{i}\right\}$ and $\left\{\tau_{i}\right\}$ and, with a walking rule appropriately chosen for that problem, a diffusion process. Also in the case of Ref. [41] the diffusion scaling does not agree with the scaling that would be produced by $\psi(t)$ if the process were renewal. The authors of Ref. [41] proposed a dynamic model with the occurrence of the observable and recordable events determined by invisible renewal events. The model proposed in [41] is quite different from the modulation model studied in the present paper, insofar as the observed events depend on the invisible events through a simple deterministic prescription. In the present case the crucial events determine a stochastic rather than deterministic prescription to generate the ordinary events. Yet, in both cases the diffusion scaling is not determined by the recorded events, but by rare renewal events behind them. On the basis of this comparison between this paper and the earlier work of Ref. [41] we are inclined to propose for the crucial events the more general definition of renewal events, visible (recorded) or invisible (not recorded), which determine the diffusion scaling coefficient. The ratio of noncrucial to crucial events is usually very small. Consequently, the noncrucial events generate a sort of camouflage action that makes it difficult to reveal the crucial events, even when they are recorded. This 
is a challenge for the search of methods of statistical analysis so efficient and sensitive as to reveal the statistical properties of rare crucial events, in spite of the camouflage action exerted by the cloud of ordinary events. The numerical experiment of aging is useful to establish whether the process is renewal or not. The assessment of the asymptotic scaling, and with it of the statistical properties of the corresponding crucial events, is a challenge to the current methods of scaling detection. We quote as an example the method of Ref. [12], which has been proven to be especially efficient. Yet, if crucial events of the kind discussed in this paper were present in real time series, an even more sensitive method of analysis would be required.

\section{ACKNOWLEDGMENTS}

P.G. thankfully acknowledges the Welch Foundation and ARO for financial support through Grants. Nos. B-1577 and W911NF-5-1-0205, respectively.
[1] S. M. Mason, Geoforum 32, 405 (2001).

[2] F. Reitsma, Geoforum 34, 13 (2003).

[3] D. C. Mikulecky, Comput. Chem. (Oxford) 25, 341 (2001).

[4] R. Metzler and T. F. Nonnenmacher, Int. J. Plast. 19, 941 (2003).

[5] G. W. Scott Blair, Nature (London) 152, 412 (1943).

[6] R. Metzler and J. Klafter, J. Non-Cryst. Solids 305, 81 (2002).

[7] G. Godréche and J. M. Luck, J. Stat. Phys. 104, 489 (2001).

[8] Eli Barkai, Phys. Rev. Lett. 90, 104101 (2003).

[9] P. Allegrini, G. Aquino, P. Grigolini, L. Palatella, and A. Rosa, Phys. Rev. E 68, 056123 (2003).

[10] P. Grigolini, in Complexity, Metastability and Nonextensivity, edited by C. Beck, G. Benedek, A. Rapisarda, and C. Tsallis (World Scientific, Singapore, 2005).

[11] P. Manneville, J. Phys. (France) 41, 1235 (1980).

[12] P. Grigolini, L. Palatella, and G. Raffaelli, Fractals 9, 439 (2001).

[13] G. Zumofen and J. Klafter, Phys. Rev. E 47, 851 (1993).

[14] S. Bianco, P. Grigolini, and P. Paradisi, J. Chem. Phys. 123, 174704 (2005).

[15] G. Aquino, M. Bologna, P. Grigolini, and B. J. West, Phys. Rev. E 70, 036105 (2004).

[16] P. Allegrini, P. Grigolini, L. Palatella, and B. J. West, Phys. Rev. E 70, 046118 (2004).

[17] P. Allegrini, P. Grigolini, L. Palatella, A. Rosa, and B. J. West, Physica A 347, 268 (2005).

[18] P. Allegrini, G. Aquino, P. Grigolini, L. Palatella, A. Rosa, and B. J. West, Phys. Rev. E 71, 066109 (2005).

[19] G. M. Zaslavsky, Phys. Rep. 371, 461 (2002).

[20] R. Verberk, A. M. van Oijen, and M. Orrit, Phys. Rev. B 66, 233202 (2002).

[21] J. P. Bouchaud, J. Phys. I 2, 1705 (1992).

[22] J. P. Bouchaud and D. S. Dean, J. Phys. I 5, 265 (1995).

[23] P. Allegrini, P. Grigolini, and A. Rocco, Phys. Lett. A 233, 309 (1997).

[24] M. Compiani, T. Fonseca, P. Grigolini, and R. Serra, Chem. Phys. Lett. 114, 503 (1985).
[25] M. F. Shlesinger and B. D. Hughes, Physica A 109, 597 (1981).

[26] E. G. D. Cohen, Physica D 193, 35 (2004).

[27] C. Beck, Phys. Rev. Lett. 87, 180601 (2001).

[28] M. Bologna, P. Grigolini, M. Pala, and L. Palatella, Chaos, Solitons Fractals 17, 601 (2003).

[29] T. Geisel, A. Zacherl, and G. Radons, Phys. Rev. Lett. 59, 2503 (1987).

[30] R. Mannella, B. J. West, and P. Grigolini, Fractals 2, 81 (1994).

[31] L. C. E. Struick, Physical Aging in Amorphous Polymers and Other Materials (Elsevier, Houston, 1978).

[32] P. Calabrese and A. Gambassi, J. Phys. A 38, R 133 (2005).

[33] W. H. Press et al., Numerical Recipes in $C++$ : The Art of Scientific Computing (Cambridge University Press, Cambridge, 2002).

[34] E. W. Montroll and G. H. Weiss, J. Math. Phys. 6, 167 (1965).

[35] P. Allegrini, J. Bellazzini, G. Bramanti, M. Ignaccolo, P. Grigolini, and J. Yang, Phys. Rev. E 66, 015101(R) (2002).

[36] P. Ehrenfest and T. Ehrenfest, Z. Phys. 27, 306 (1926).

[37] G. Cattaneo, Atti Semin. Mat. Fis. Univ. Modena 3, 83 (1948).

[38] B. V. Gnedenko and A. N. Kolmogorov, Limit Distributions for Sums of Independent Random Variables (Addison-Wesley, Reading, MA, 1954).

[39] E. W. Montroll and B. J. West, in Studies in Statistical Mechanics Vol. VII: Fluctuation Phenomena, edited by E. W. Montroll and J. L. Lebowitz (North-Holland, Amsterdam, 1979).

[40] I. S. Gradshtein and I. M. Ryzhik, Table of Integrals, Series, and Products (Academic, San Diego, 1980), p. 317.

[41] P. Allegrini, P. Grigolini, P. Hamilton, L. Palatella, and G. Raffaelli, Phys. Rev. E 65, 041926 (2002).

[42] M. Kuno, D. P. Fromm, H. F. Hamann, A. Gallagher, and D. J. Nesbitt, J. Chem. Phys. 115, 1028 (2001).

[43] X. Brokmann, J.-P. Hermier, G. Messin, P. Desbiolles, J.-P. Bouchaud, and M. Dahan, Phys. Rev. Lett. 90, 120601 (2003). 